\title{
Ten-Year obesity and overweight prevalence in Greek children: A systematic review and meta-analysis of 2001-2010 data
}

\author{
Eleni P. Kotanidou, ${ }^{1}$ Maria G. Grammatikopoulou, ${ }^{2}$ Bessie E. Spiliotis, ${ }^{3}$ \\ Christina Kanaka-Gantenbein, ${ }^{4}$ Maria Tsigga, ${ }^{2}$ Assimina Galli-Tsinopoulou ${ }^{1}$
}

${ }^{1} 4^{\text {th }}$ Department of Pediatrics, Faculty of Medicine, Aristotle University of Thessaloniki, ${ }^{2}$ Department of Human Nutrition and Dietetics, Alexander Technological Educational Institute of Thessaloniki, Thessaloniki, ${ }^{3}$ Division of Pediatric Endocrinology and Diabetes, Department of Pediatrics, School of Medicine, University of Patras, Patras, ${ }^{4}$ Division of Endocrinology, Diabetes and Metabolism, First Department of Pediatrics, Faculty of Medicine, University of Athens, Athens, Greece

\begin{abstract}
OBJECTIVE: While the US today has the highest rates worldwide of obesity, Europe, and particularly Southern Europe, is catching up fast. The aim of this study was to report the prevalence of obesity in Greek children, aged 1-12 years. DESIGN: A systematic review - including all studies published in English and Greek from January 2001 until December 2010 regarding childhood obesity, using the IOTF criteria - was performed. Twenty-five out of 134 published studies were finally selected, including 219,996 boys and 210,772 girls. RESULTS: Meta-analysis revealed that 10.2\% (CI 95\%: 9.8-10.7\%) of Greek children (1-12 years) are obese, $23.7 \%$ (CI 95\%: 22.7-24.8\%) are overweight and the combined prevalence of overweight and obesity is 34\% (CI 95\%: 32.7-35.3\%). Subgroup analysis by gender showed that $11 \%$ of the boys and $9.7 \%$ of the girls were obese, while $24.1 \%$ of the boys and $23.2 \%$ of the girls were overweight. The combined prevalence of excess in body weight predominated in boys (35\%), while in girls the above prevalence was $32.7 \%$. Cumulative analysis revealed an upward trend of the phenomenon (2001-2003), followed by a stabilization (2003-2010). CONCLUSIONS: During the decade 2001-2010, 1/10 Greek children was obese and 3/10 were overweight. The implementation of policies to reverse childhood obesity is of the utmost urgency.
\end{abstract}

Key words: Children, Europe, Greece, Meta-analysis, Obesity, Overweight, Prevalence

Address for correspondence:

Assimina Galli-Tsinopoulou, $4^{\text {th }}$ Department of Pediatrics, Faculty of Medicine, Aristotle University of Thessaloniki, Papageorgiou General Hospital, Ring Road Nea Efkarpia, GR 56403 Thessaloniki, Greece; Tel./Fax: +30 2310991537; e-mail: gallitsin@gmail.com \& galtsin@otenet.gr Received 16-04-2012, Accepted 09-10-2012

\section{INTRODUCTION}

Three elements appear to contribute to the development of the obesogenic environment prevalent in the Western world but also rising fast in developing countries: food availability, urbanization and sedentary lifestyle. Their both independent and synergistic 
interactions have resulted in the obesity epidemic, contributing to 2.6 million deaths worldwide on an annual basis. ${ }^{1}$ Childhood and adolescence have been deemed critical periods for the development of the condition, since approximately one half of overweight adolescents and over one third of overweight children become obese adults, all with an increased risk for premature death during early adult life. ${ }^{2,3}$ Additionally, obesity is hallmarked by a plethora of comorbidities, all contributing to an increased health care economic cost, especially in youth aged 6-17 years. ${ }^{4}$

With regard to Europe, according to recent data there is wide geographical variation across European countries of obesity and overweight rates among children and adolescents. ${ }^{5}$ The over-time trend of the phenomenon is uneven, following an augmentative trend in some countries, while in others there is evidence that the rates have leveled off. 5,6

One of the first arrows in the quiver of health care providers and policy formations is appropriate research assessing the epidemiologic transitions of childhood overweight and obesity at both a national and an international level. The accurate assessment and classification of the obesity phenomenon will enable recognition and understanding of the epidemiology, this leading to high quality data describing the problem and determining the burden of disease.

Different protocols focusing on the problem of pediatric obesity in Greece have been implemented during the last few years. Previously published reviews have sought to elucidate the phenomenon in a national context. However, to date no research has integrated the outcomes reported from all different available epidemiological protocols and reviews from a meta-analytic perspective.

The aims of this study were a) to provide an accurate overview of the recent relevant data by performing a systematic review of the last ten years' literature and b) to conduct a meta-analysis of the best evidence available (meta-analysis of prevalence rates) in order to determine the current extent of the problem and to estimate the over-time trend of childhood obesity.

\section{MATERIALS AND METHODS}

Meta-analysis is a well-established method in medical research, used to combine and analyze large collections of previous research outcomes in order to arrive at a clear scientific hypothesis. ${ }^{7}$ Meta-analytic approaches are typically, but not necessarily, applied to randomized controlled trials and generally require methodological homogeneity of the studies included to assess the outcome of a treatment or the effect of a risk factor in any particular pathology. However, the present meta-analysis combines methodologically highly heterogeneous protocols to synthesize and meta-analyze prevalence rates reported from different samples of a national childhood population. This type of meta-analysis has previously been developed and widely used to estimate the prevalence of a specific phenomenon/disease. ${ }^{8,9}$

\section{Search strategy}

The study was designed according to the Metaanalysis of Observational Studies in Epidemiology group guidelines for reporting meta-analysis of observational studies. ${ }^{10}$ Two of the investigators (E.P.K. and M.G.G.) collected all available data on the prevalence of pediatric overweight $(\mathrm{OW})$ and obesity $(\mathrm{OB})$ in Greece, published from January 2001 until December 2010. A literature search was conducted in MEDLINE, EMBASE, SCOPUS, Web of Science and Google Scholar, during October-November 2010, and updated again in January 2011. The search terms were: "children”, “childhood”, “obesity”, “overweight”, "weight status", "body composition", "Greece", "Greek".

In order to avoid language publication bias, an additional literature search was performed using the abovementioned terms in the Greek language via the two national scientific search engines Iatrotek Online and Openarchives.gr as well as the electronic database of Google.gr. Furthermore, a search in the electronic versions of the two Greek pediatric journals through the links www.e-child.gr and www.paediatriki. $g r$ was performed. The reference lists of all retrieved manuscripts were checked for additional data.

In cases where relevant data were missing or it was not possible to obtain full-text papers, the corresponding authors were contacted via e-mail for additional information. The search procedure yielded 134 publications, published between January 2001 and December 2010. 


\section{Eligibility of relevant studies}

To identify studies eligible for the meta-analysis, two of the reviewers independently screened all collected papers on the basis of their abstract and fulltext. In the event of disagreement, a consensus was reached after the consultation of a third independent investigator.

Research reporting the prevalence of $\mathrm{OW} / \mathrm{OB}$ in Greek children was considered relevant only when the following criteria were met: (a) definition of OW and OB according to the International Obesity Task Force (IOTF) criteria, ${ }^{11}$ (b) studies included pre-pubertal children aged up to the age of 12 years (the age cutoff was based on the educational unit format of Greece, since the majority of the identified studies selected school-based samples) and (c) published between January 2001-December 2010. Studies were excluded from the analyses when: (a) they reported only mean BMI values without investigating the prevalence of $\mathrm{OW} / \mathrm{OB},(\mathrm{b})$ they did not segregate data into gender group, (c) they referred to data collected prior to the year 2000, to prevent systematic bias, or (d) if they referred to apparently non-healthy children. When one population was reported in more than one publication, only the most recent one or the one providing the maximum of information was included to avoid sample overlapping.

\section{Data coding and classification}

A predefined standard information extraction sheet was used in order to solicit data from each eligible study. Study general profile (authors, year, journal, design, study name, setting), methodology (measurement, sample collection, time of data collection), sample characteristics (size, age range, source, geographical origination, rural/urban, response rate), outcomes (prevalence of $O W / O B$ in the total sample and according to gender) were extracted from each publication. When a study reported the prevalence of childhood OW/OB for more than one population separately or for more than one data collection time milestones - e.g. for 6-year-old and 12-year-old participants separately or prevalence measured in 2002 and prevalence measured in 2004 - these subsamples were classified as two discrete study populations and treated as independent.

\section{Quality assessment of studies}

In order to assess quality of the eligible studies and to control for possible bias, specific study characteristics suggested by Lien et al. and the criteria proposed in the Newcastle-Ottawa Scale for nonrandomized studies were selected, adopted and applied. ${ }^{5,12}$ The final scoring system (Table 1) comprised 9 criteria rating different quality elements for each eligible paper. Authors recommended scale weights

Table 1. Items used to measure quality of the selected studies

\begin{tabular}{llc}
\hline Quality item & & Points \\
\hline Sample size (n) & $\leq 500 / 501-1000 / 1001-2000 / 2001-3999 / \geq 4000$ & $0 / 0.5 / 1 / 1.5 / 2$ \\
Geographic area of origination & Local/National & $0 / 1$ \\
Sample Source Methodology (School sample/ Randomized sample) & Reported/NR & $1 / 0$ \\
Sample Unit Selection Methodology (All units/ Randomized units) & Reported/NR & $1 / 0$ \\
Participants Selection Methodology (All participants of each unit/ & Reported/NR & $1 / 0$ \\
Randomization of participants in each unit) & & $1 / 0$ \\
Data Collection Time & Reported/NR & $1 / 0.5 / 0 / 0$ \\
Adequate Response Rate & $\geq 75 \% / 61-75 \% / \leq 60 \% / N R$ & $1 / 0 / 0$ \\
Urban/Rural Sample & Urban + Rural sample/ Isolated Urban/ & \\
& Isolated Rural & $2 / 0 / 0$ \\
Data Measurement Methodology & Data measured by the investigators/ Data & \\
Reporting outcome per age/per year of birth & reported/NR & $1 / 0$ \\
& Yes/No & 12 points \\
\hline
\end{tabular}

NR: Not Reported. 
for each element of the scoring system, as proposed in other meta-analyses. Studies were classified into three quality groups and labeled $A$ if they managed to obtain 8-12 points, B when 4-7 points were collected and $\mathrm{C}$ when they scored less than 4 points. Studies of high quality, with less estimated risk of bias, were allocated to class A and were subsequently used in the sensitivity analyses.

\section{Assessment of heterogeneity}

The null hypothesis of the existence of homogeneity in the prevalence reported in the studies was tested with I ${ }^{2}$ and Cochrane's Q. ${ }^{13,14}$ Cochrane's Q statistic, an approximate to the $\mathrm{x}^{2}$ test of heterogeneity, with $\mathrm{k}-1$ degrees of freedom where $\mathrm{k}$ is the number of prevalence rates, was tested in an a-level of $0.10 .{ }^{13}$ $\mathrm{I}^{2}$ describes the total variation across studies and was used to identify violation in the assumption of homogeneity when $\mathrm{I}^{2} \geq 75 \% .^{14}$

\section{Statistical Methods}

Comprehensive Meta Analysis version 2.0 (Comprehensive Meta Analysis ${ }^{\odot}$, Biostat, Inc Englewood, USA) served as the statistical platform. The effect size of the meta-analysis was the prevalence of childhood OW/OB, computed - when not referred to - as the ratio of the events (number of $\mathrm{OW}$ or OB participants)/total (total number of participants). Effect size was pooled from all eligible studies using the DerSimonian-Laird Random effects model (REM) for meta-analysis. ${ }^{15}$ This model was preferred to a fixed effect model, since it is based on the assumption that a distribution of effects exists, resulting in heterogeneity among study results. ${ }^{7}$ For space economy, results are presented in tables and forest plots where prevalence rates and $95 \%$ confidential intervals (CI) are figured out for every study inserted in the model and for the overall estimate.

To retrieve the extent of publication bias, funnel plots were scattered and tested for asymmetry and, additionally, Egger's test was computed. Subgroups analysis was conducted for each gender. The effect of aberrant studies was examined via sensitivity analyses after the exclusion of studies with low and poor quality, as classified by the quality scoring system (B and $\mathrm{C}$ class studies). Over-time trend in the prevalence of $\mathrm{OW} / \mathrm{OB}$ was estimated via cumulative analyses which calculate the cumulative evidence at the time of the appearance of each data set. To avoid systematic bias, studies were entered into the model of each cumulative meta-analysis successively according to the data collection time and not to the publication time. Publications not reporting the year of data collection were not included in the cumulative analyses.

\section{RESULTS}

\section{Literature records selection}

The search yielded 167 studies, 34 of which were excluded at the first step on the basis of their title, since it was considered irrelevant to the research hypothesis (Figure 1). Of the remaining 133 studies, 36 were omitted because of reporting of duplicate data and use of overlapping samples. The remaining 97 studies underwent discrete examination by two independent reviewers. Thirteen studies were excluded due to defining OW/OB differently from the IOTF criteria, 4 studies due to grouping OW and OB participants together and 14 studies because of failure to report the prevalence separately in each sex. Furthermore, 28 of the retrieved publications either failed to report the prevalence of $\mathrm{OW} / \mathrm{OB}$ or used pediatric samples with excess in body weight only, thus omitting to calculate prevalence. Moreover, one study was excluded because of reference to non-phenotypically healthy children and 3 additional publications were excluded due to collection of data prior to the year 2001. Of the remaining records, 9 studies that examined the prevalence in children and adolescents older than 12 years were also excluded. Overall, 109 publications did not meet inclusion criteria and thus only 25 of the retrieved studies were used in the meta-analyses. ${ }^{6,16-39}$

\section{Characteristics of studies included in the analyses}

Evidence extracted from the 25 eligible studies consisted of epidemiological data published in Greek and international peer-reviewed journals during 20042010 (Table 2). They reported the rates of OW/OB from 31 different pediatric populations, since two publications included more than one sample collected during multiple cross-sectional wave recruitments, ${ }^{6,20}$ which were treated separately. A subscript after the reference was used to distinguish samples. 


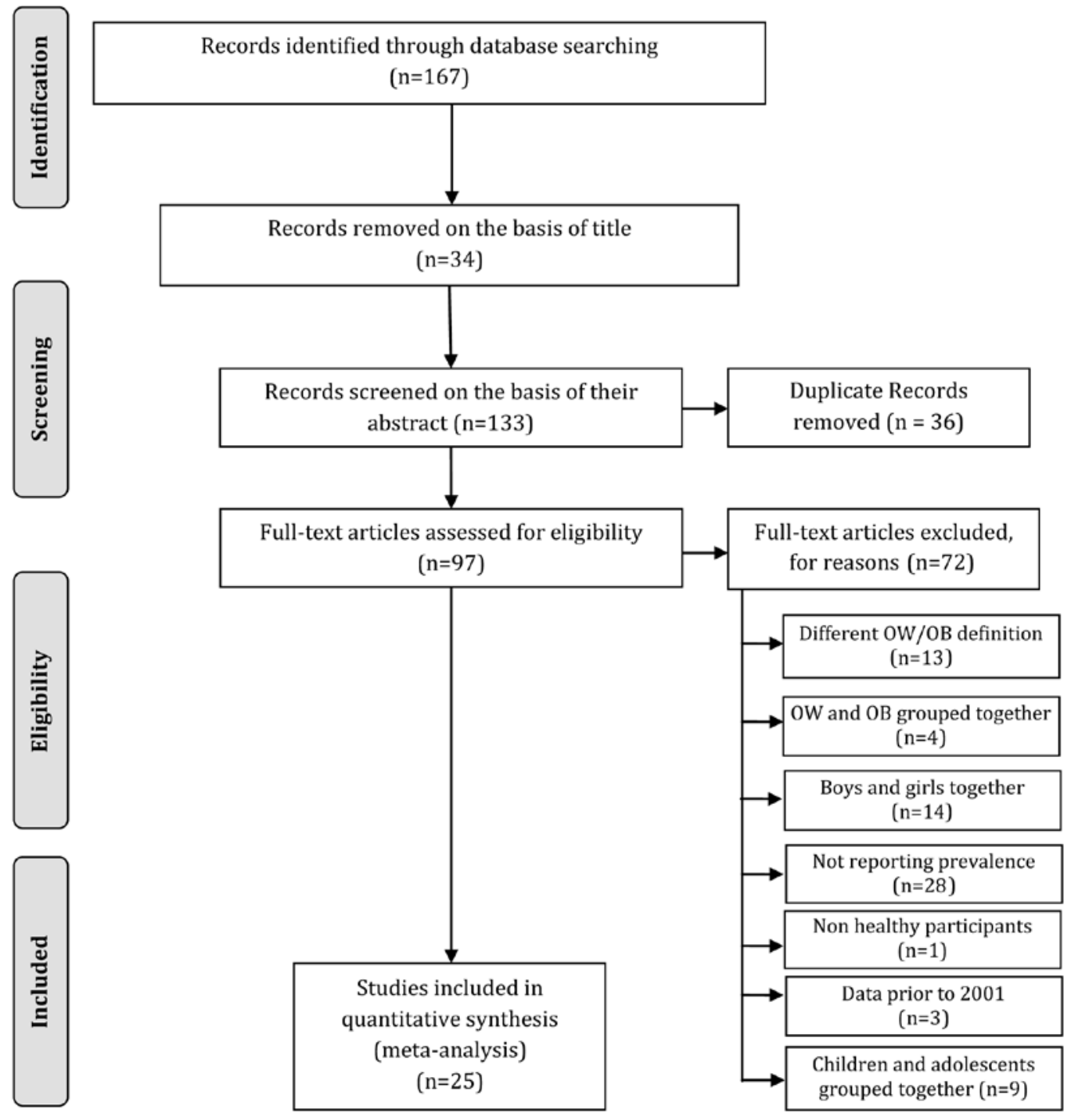

Figure 1. Flow chart of the selection process for the studies included in the analyses.

Thus, 31 different studies covering a total of 430,768 Greek children, 219,996 boys and 210,772 girls, were used for the meta-analyses. Sample size ranged from 73 to 71,227 participants, inhabitants of the mainland as well as of the Greek islands. The majority (28/31) were school-based samples aiming at detecting the prevalence of excess in body weight among Greek pupils. Descriptive characteristics and quality scoring of the studies are presented in detail in Table 2.

\section{Main analyses - Prevalence of $O W$ and $O B$}

Prevalence of OW and OB in the whole sample and according to gender for each analysis is presented in Table 3. Obesity in Greek children during the last decade is reported to range from 3.35-23.29\%. ${ }^{17,27}$ However, since the studies were found to be highly heterogeneous $\left(\mathrm{Q}=415.8 \mathrm{p}<0.001, \mathrm{I}^{2}=92.8 \%\right)$, a REM was applied. The overall rate of obesity prevalence in Greek children was estimated at $10.2 \%$ (CI 95\%: 9.8-10.7\%). The funnel plot appeared quite symmetrical but Egger's test revealed evidence of publication bias (Egger's test $\mathrm{p}=0.045$ ). The prevalence of OW ranged from 12.1-34.3\% ${ }^{18,35}$ among the selected studies. Meta-analysis yielded an overall pooled prevalence of $23.7 \%$ (CI 95\%: 22.7-24.8\%) but increased heterogeneity was demonstrated among the studies $\left(\mathrm{Q}=1355.3, \mathrm{p}<0.001, \mathrm{I}^{2}=97.8 \%\right)$. Fun- 


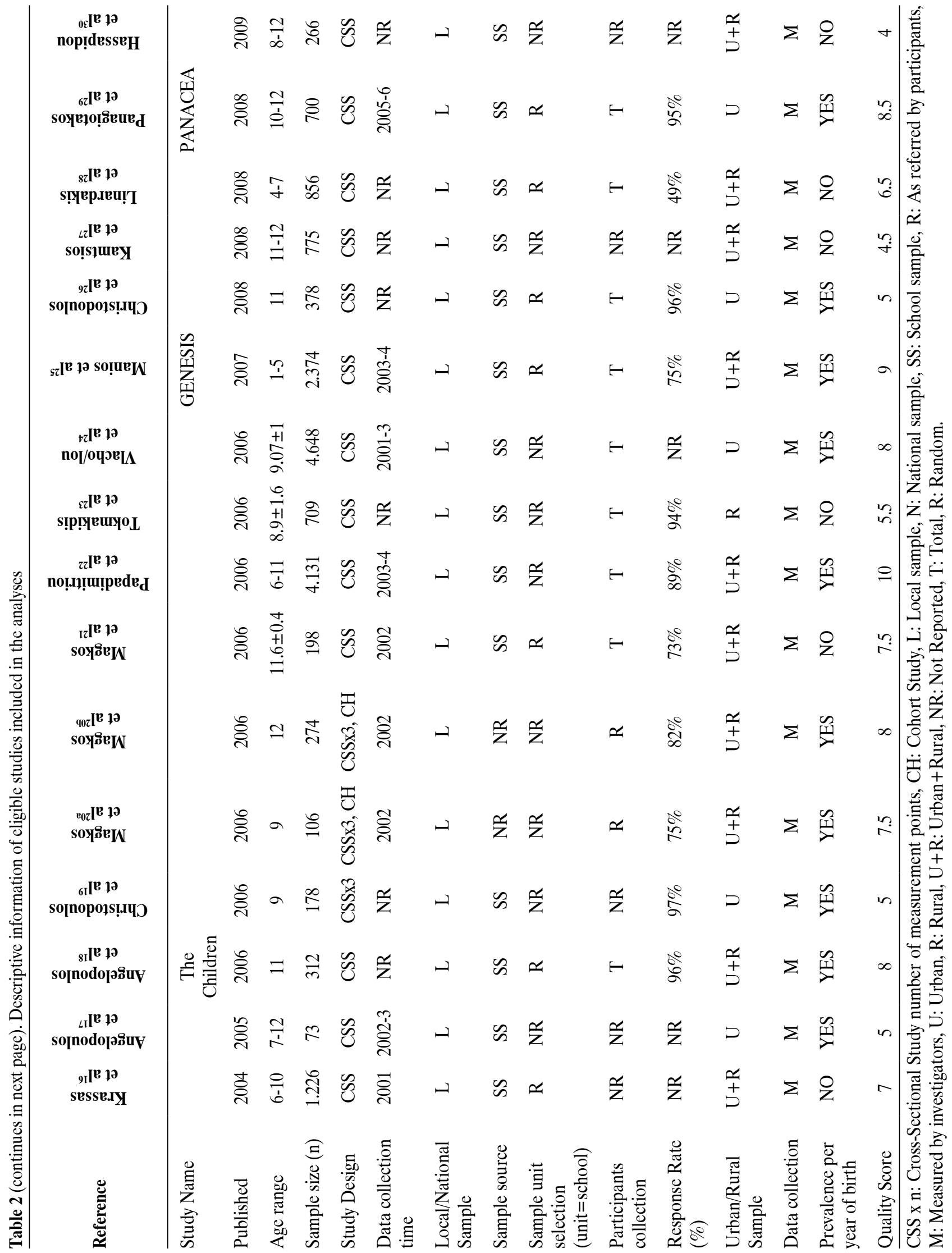




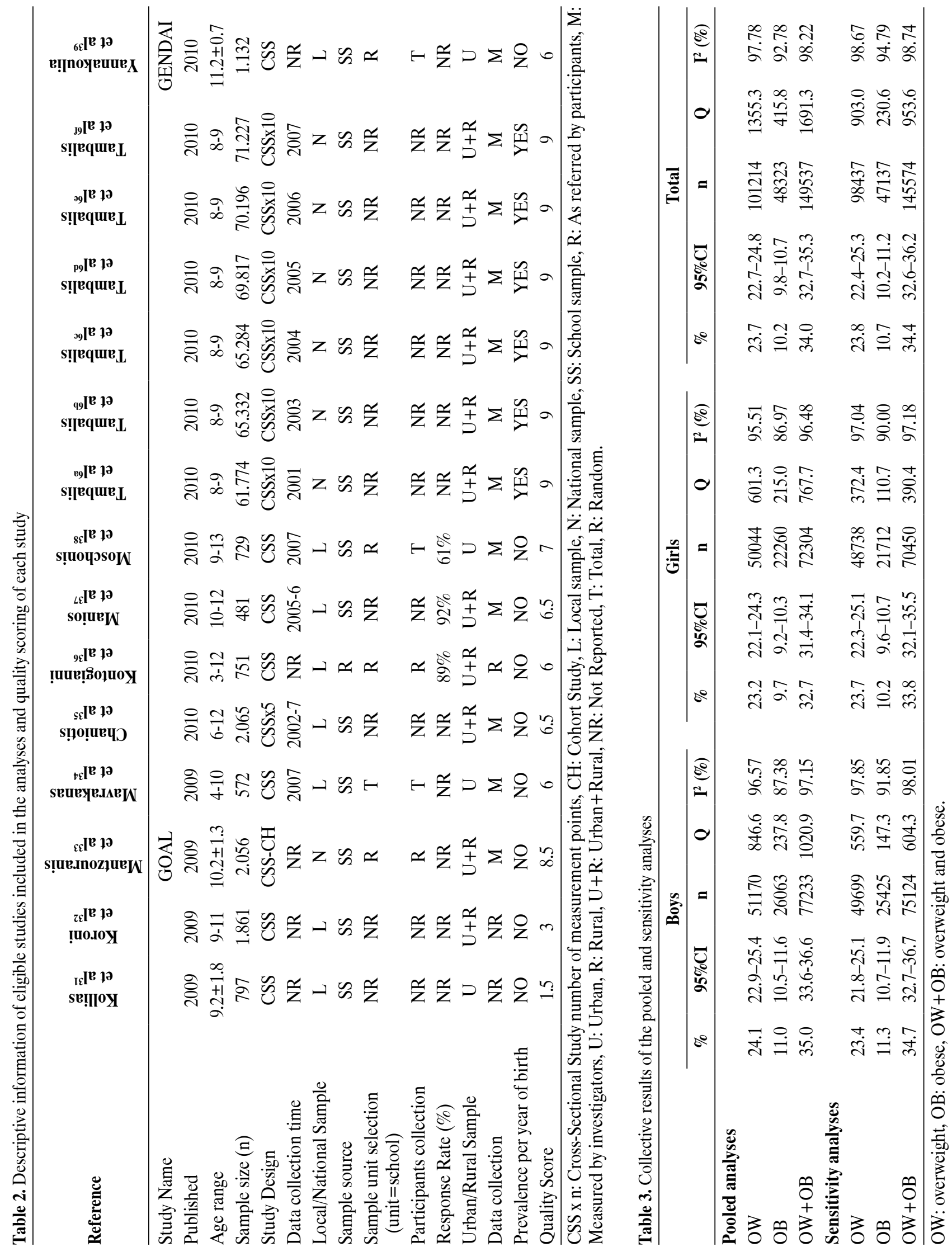


nel plot symmetry in accordance with Egger's test $(p=0.95)$ suggested lack of publication bias. The combined pooled prevalence of $\mathrm{OW}$ and $\mathrm{OB}$ ranged from $17.63-56.16 \%,{ }^{17,35}$ but the sample was not homogenous $\left(\mathrm{Q}=1691.3, \mathrm{p}<0.001, \mathrm{I}^{2}=98.3 \%\right)$. The overall combined prevalence of OW and OB in Greek children reached 34\% (CI 95\%: 32.7-35.3\%), without evidence of publication bias (Egger's test $\mathrm{p}=0.54$ ).

\section{Subgroups}

The prevalence of OB ranged from $2.5-23.1 \%{ }^{17,27}$ in the boys and between 4.1-23.5\% ${ }^{17,27}$ in the girls. The prevalence of OW ranged from $11.69-35.87 \% \%^{35,39}$ in the boys and $12.50-38.99 \% \%^{18,35}$ in the girls. Subgroups gender analysis failed to determine possible sources of heterogeneity among the studies. Eleven percent of the boys [CI 95\%: 10.5-11.6\%, Q =237.8 p<0.001, $\mathrm{I}^{2}=87.4 \%$ ] and $9.7 \%$ of the girls [CI 95\%: 9.2-10.3\%, $\mathrm{Q}=214.9 \mathrm{p}<0.001, \mathrm{I}^{2}=86.9 \%$ ] were classified as OB. Overweight was apparent in $24.1 \%$ of the boys [CI 95\%: $\left.22.9-25.4 \%, \mathrm{Q}=846.6 \mathrm{p}<0.001, \mathrm{I}^{2}=96.6 \%\right]$, and $23.2 \%$ of the girls [CI 95\%: $22.1-24.3 \%, \mathrm{Q}=601.3$ $\left.\mathrm{p}<0.001, \mathrm{I}^{2}=95.5 \%\right]$. Combined prevalence of OW and OB was $35 \%$ in the boys [CI 95\%: 33.6-36.6\%, $\left.\mathrm{Q}=1020.9 \mathrm{p}<0.001, \mathrm{I}^{2}=97.2 \%\right]$ and $32.7 \%$ in the girls [CI 95\%:31.4-34.1\%, Q $=767.6 \mathrm{p}<0.001, \mathrm{I}^{2}=96.5 \%$ ).

\section{Sensitivity analyses}

Sensitivity analyses were performed for all of the studied effect sizes (OW, OB and combined prevalence rates) by removing data from the meta-analytic model in order to examine influence of low quality and high-bias-risk studies on the overall estimate. According to the quality score (Table 2), only studies of higher quality $(n=13,41.9 \%)$ were included in the sensitivity analyses. The sensitivity analyses yielded an OB prevalence of $10.7 \%$ [CI 95\%: 10.2-11.2\%, $\left.\mathrm{Q}=230.6 \mathrm{p}<0.001, \mathrm{I}^{2}=94.8 \%\right]$ and an $\mathrm{OW}$ rate equal to $23.8 \%$ [CI 95\%: $22.4-25.3 \%, \mathrm{Q}=902.9 \mathrm{p}<0.001$, $\mathrm{I}^{2}=98.7 \%$ ]. Combined prevalence of $\mathrm{OW}$ and $\mathrm{OB}$ was $34.4 \%$ [CI 95\%: 32.6-36.2\%, Q=953.6 p $<0.001$, $\mathrm{I}^{2}=98.7 \%$ ]. Gender analysis revealed that among the boys $11.3 \%$ were diagnosed with OB [CI 95\%: $\left.10.7-11.9 \%, \mathrm{Q}=147.3 \mathrm{p}<0.001, \mathrm{I}^{2}=91.9 \%\right)$ and $23.4 \%$ were OW [CI 95\%: 21.8-25.1\%, Q =559.7 p <0.001, $\left.\mathrm{I}^{2}=97.9\right]$. In the girls' group $10.2 \%$ were OB [CI 95\%: 9.6-10.7\%, Q=110.7 $\left.\mathrm{p}<0.001, \mathrm{I}^{2}=90 \%\right)$ and 23.7\% were OW [CI 95\%: 22.3-25.1\%, Q=372.4 $\mathrm{p}<0.001, \mathrm{I}^{2}=97 \%$ ]. Combined prevalence of OW and OB was estimated for the boys at $34.7 \%$ [CI 95\%: $\left.32.7-36.7 \%, \mathrm{Q}=604.3 \mathrm{p}<0.001, \mathrm{I}^{2}=98 \%\right]$ and at $33.8 \%$ for the Greek girls [CI 95\%: 32.1-35.5\%, $\left.\mathrm{Q}=390.4 \mathrm{p}<0.001, \mathrm{I}^{2}=97.2 \%\right]$.

\section{Cumulative meta-analyses}

Cumulative meta-analyses forest plots confirmed the upgrading trend of the OB prevalence during the first 3 years of the decade (2001-2003) and provided evidence for the rates' stabilization trend during the last 5 years. Similar evolutionary patterns (leveling off and stabilizing thereafter) were also exhibited when cumulative meta-analyses were run for each gender separately (Figure 2).

\section{DISCUSSION}

The prevalence of $\mathrm{OW}$ and $\mathrm{OB}$ in Greek children appears alarming, with approximately $1 / 3$ of the children demonstrating excess in body weight. During the studied decade 2001-2010, the rate of OB increased and appears to have reached a plateau some time during the year 2005. No data collected after 2007 were found in the literature or included in the analyses, therefore it is difficult to approximate the latest trend of the condition in the Greek pediatric population.

Both genders exhibited a peak in the prevalence of OB during the year 2005. As OB is also socially mediated, it is possible that the 2004 Olympics that were hosted in Greece served as a motive for increasing physical activity and participation in sports, as the number of OB children appears to have leveled off after the event. It is also likely that the characterization of $\mathrm{OB}$ as an epidemic, which took place during the early 2000's, ${ }^{40}$ alarmed Greek parents. According to Rokholm et al, ${ }^{41}$ stabilization, leveling off or a decrease in the prevalence of pediatric OB has been noted in Europe since the year 1999. Prior to the year 1999, the number of OW and OB children was significantly lower in Greece and, according to Manios et al, ${ }^{42}$ a threefold increase was noted in 2005 compared to the 1980's. Tambalis and colleagues ${ }^{6}$ demonstrated an augmentation in the prevalence of pediatric OB during 1997-2004, followed by a leveling off in the ensuing 2004-2007 period, for both sexes. It is possible that the stabilization observed by Tambalis stems from the fact that only children of a particular 
(a)

\begin{tabular}{|c|c|c|c|c|c|c|}
\hline \multirow[t]{2}{*}{ Authors } & \multirow[t]{2}{*}{$\underline{\text { References }}$} & \multirow[t]{2}{*}{ Time point } & \multicolumn{3}{|c|}{ Cumulative statistics } & \multirow[b]{2}{*}{ Total } \\
\hline & & & Point & Z-Value & p-Value & \\
\hline Krassas et al. & 16 & 2001 & 0.065 & -16.123 & 0.000 & $39 / 602$ \\
\hline Tambalis et al. (a) & $6 a$ & 2001 & 0.105 & -117.657 & 0.000 & $3383 / 32152$ \\
\hline Magkos et al. (II) & 21 & 2002 & 0.105 & -117.819 & 0.000 & $3389 / 32244$ \\
\hline Magkos et al (la) & $20 a$ & 2002 & 0.105 & -118.005 & 0.000 & $3402 / 32350$ \\
\hline Magkos et al. (Ib) & $20 b$ & 2002 & 0.105 & -118.492 & 0.000 & $3434 / 32624$ \\
\hline Angelopoulos et al. & 17 & 2003 & 0.106 & -118.509 & 0.000 & $3443 / 32663$ \\
\hline Vlachopapadopoulou et al. & 24 & 2003 & 0.105 & -122.776 & 0.000 & $3672 / 35028$ \\
\hline Tambalis et al. (b) & $6 b$ & 2003 & 0.112 & -170.830 & 0.000 & $7678 / 68688$ \\
\hline Manios et al. (I) & 25 & 2004 & 0.111 & -172.271 & 0.000 & $7754 / 69906$ \\
\hline Papadimitriou et al & 22 & 2004 & 0.112 & -174.729 & 0.000 & $8006 / 71960$ \\
\hline Tambalis et al. (c) & $6 c$ & 2004 & 0.115 & -210.499 & 0.000 & $12087 / 105135$ \\
\hline Tambalis ot al. (d) & $6 d$ & 2005 & 0.118 & -242.736 & 0.000 & $16456 / 140369$ \\
\hline Manios et al. (II) & 37 & 2006 & 0.118 & -242.912 & 0.000 & $16491 / 140600$ \\
\hline Panagiotakos et al. & 29 & 2006 & 0.118 & -243.197 & 0.000 & $16519 / 140923$ \\
\hline Tambalis et al. $(\theta)$ & 6 & 2006 & 0.119 & -272.148 & 0.000 & $20930 / 176786$ \\
\hline Mavrakanas et al. & 34 & 2007 & 0.119 & -272.358 & 0.000 & $20969 / 177073$ \\
\hline Moschonis et al. & 38 & 2007 & 0.119 & -272.614 & 0.000 & $21027 / 177452$ \\
\hline Chaniotis et al. & 35 & 2007 & 0.119 & -273.331 & 0.000 & $21085 / 178453$ \\
\hline \multirow[t]{2}{*}{ Tambalis et al. (f) } & $6 \mathrm{f}$ & 2007 & 0.119 & -299.821 & 0.000 & $25525 / 214849$ \\
\hline & & & 0.119 & -299.821 & 0.000 & \\
\hline
\end{tabular}

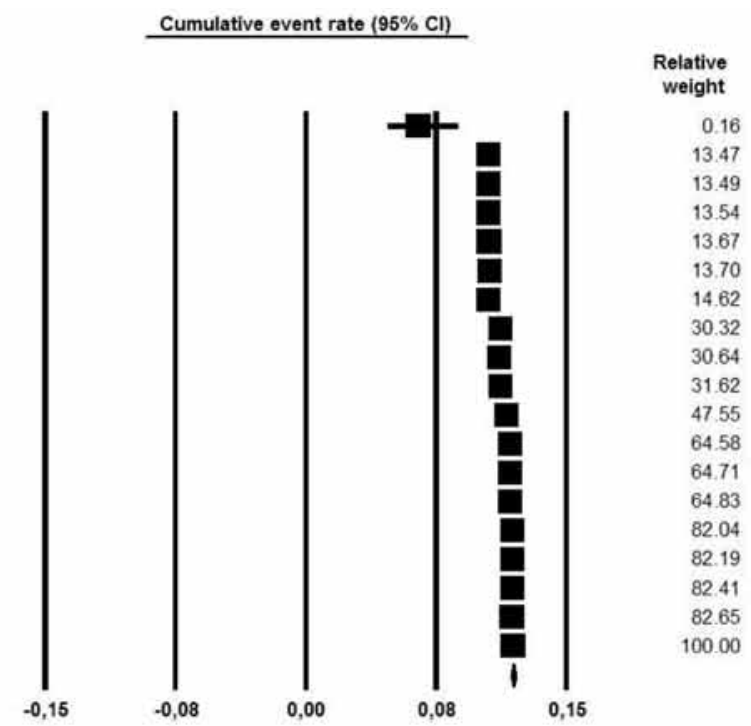

(b)

\begin{tabular}{|c|c|c|c|c|c|c|}
\hline \multirow[t]{2}{*}{ Authors } & \multirow[t]{2}{*}{ References } & \multirow[t]{2}{*}{ Time point } & \multicolumn{3}{|c|}{ Cumulative statistics } & \multirow[b]{2}{*}{ Total } \\
\hline & & & Point & Z-Value & p-Value & \\
\hline Krassas et al. & 16 & 2001 & 0.050 & -16.018 & 0.000 & $31 / 624$ \\
\hline Tambalis et al. (a) & $6 a$ & 2001 & 0.092 & -116.050 & 0.000 & $2842 / 30848$ \\
\hline Magkos et al. (II) & 21 & 2002 & 0.092 & -116.244 & 0.000 & $2849 / 30954$ \\
\hline Angelopoulos et al. & 17 & 2003 & 0.093 & -116.249 & 0.000 & $2857 / 30988$ \\
\hline Vlachopapadopoulou et al. & 24 & 2003 & 0.092 & -120.474 & 0.000 & $3046 / 33271$ \\
\hline Tambalis et al. (b) & $6 b$ & 2003 & 0.099 & -167.689 & 0.000 & $6402 / 64933$ \\
\hline Papadimitriou et al. & 22 & 2004 & 0.099 & -170.362 & 0.000 & $6607 / 67010$ \\
\hline Manios et al. (I) & 25 & 2004 & 0.099 & -171.834 & 0.000 & $6701 / 68166$ \\
\hline Tambalis et al. (c) & $6 c$ & 2004 & 0.103 & -207.708 & 0.000 & $10329 / 100275$ \\
\hline Tambalis et al. (d) & $6 d$ & 2005 & 0.105 & -240.304 & 0.000 & $14044 / 134358$ \\
\hline Panagiotakos et al. & 29 & 2006 & 0.105 & -240.646 & 0.000 & $14078 / 134735$ \\
\hline Manios et al. (II) & 37 & 2006 & 0.105 & -240.871 & 0.000 & $14103 / 134985$ \\
\hline Tambalis et al. (e) & 6 & 2006 & 0.105 & -269.789 & 0.000 & 17777 / 169318 \\
\hline Mavrakanas et al. & 34 & 2007 & 0.105 & -269.987 & 0.000 & $17818 / 169603$ \\
\hline Moschonis et al. & 38 & 2007 & 0.105 & -270.257 & 0.000 & $17841 / 169953$ \\
\hline Tambalis et al. (f) & $6 f$ & 2007 & 0.106 & -296.438 & 0.000 & $21742 / 204784$ \\
\hline \multirow[t]{2}{*}{ Chaniotis et al. } & 35 & 2007 & 0.106 & -297.133 & 0.000 & $21798 / 205848$ \\
\hline & & & 0.106 & -297.133 & 0.000 & \\
\hline
\end{tabular}

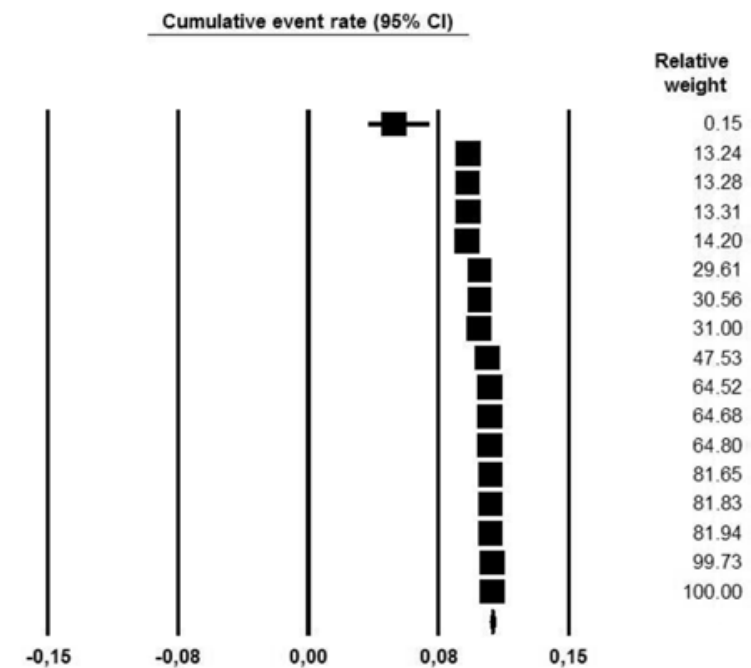

Figure 2. Cumulative analyses of the prevalence of $O B$ in Greek boys (a) and girls (b).

age ( 8 years old only) were included in the study and not a wider age-range sample. In any case, pediatric OW rates were reported as continuously rising, for both boys and girls. ${ }^{6}$

Georgiadis and Nassis ${ }^{43}$ studied the 1990-1991 data of a nationwide sample of Greek youngsters aged 6-17 year-old and found that the prevalence of $\mathrm{OB}$ was comparable to previously reported rates from Greece, as well as to rates originating from most European countries at the same period. When the sample was divided by gender, the number of OW children demonstrated an irregular augmentation in the boys and a non-linear decrease in the girls. The finding was attributed to the dieting habit frequently adopted by girls when entering adolescence. ${ }^{43} \mathrm{De}$ spite the guidelines for reporting the prevalence of OW/OB by year of birth, ${ }^{44}$ the majority of studies retrieved used wide age samples and did not report the prevalence of each age. Thus, it is difficult to obtain an accurate image of the pediatric OW and $\mathrm{OB}$ age trends in Greece. However, since the BMI at 6 years of age comprises a good indicator of BMI during adulthood, ${ }^{45}$ the situation in Greece appears dire and everything strongly indicates that now is the 
right time for intervention in order to sustain health in the future adult populations.

Europe appears to be a multi-leveled pattern area regarding prevalence rates in the childhood obesity epidemic. The European North exhibits sustained levels of childhood weight disorders, since in 7-9 yearold Swedish children only $15.6 \%$ are OW, including $2.6 \%$ OB.${ }^{46}$ Likewise, in Norway the rate of primary school children with excess in body weight is $17 \% .{ }^{47}$ In England the overweight and obesity trend have stabilized in recent years; odds ratio for being an OW or OB child in UK has leveled off $(\mathrm{OR}=0.99)$ or remained the same $(\mathrm{OR}=1.06)$, respectively, when comparing data of 2002-3 to 2006-7.48

On the other hand, the European South presents alarming rates of $\mathrm{OW}$ and $\mathrm{OB}$ over the last decade. In Spain, $10.3 \%$ of children aged $2-15$ year-old are OB and $18.8 \%$ are $\mathrm{OW},{ }^{49}$ a result similar to the present study where approximately $1 / 3$ of Greek children demonstrated excess in body weight. Combined prevalence of OW and OB reaches $28.1 \%$ in Portuguese children aged 7-9 years old. ${ }^{50}$ It is uncertain if the current economic crisis will reduce the phenomenon of hyperphagia or if it will actually refuel obesity by increasing the consumption of cheap fat/sugar-dense foods. However, in Italy, another European country affected by the economic crisis, a slight decrease has been noted in childhood overweight during 2010-11 when compared to the beginning of the 2000's. ${ }^{51}$

Obesity during childhood is a complex phenomenon attributed to both nature (genetics) and nurture (behavior). ${ }^{52}$ Diet appears to be an important behavioral factor in the development of childhood OW. "High fiber and low sugar-added beverages" and "dinner, cooked meals and vegetables pattern" are dietary patterns negatively associated with all obesity indices in Greek children. ${ }^{39}$ Kindergarten children in Crete who consume sugar-added beverages frequently demonstrated double odds in being OW/OB, ${ }^{28}$ while Greek childhood BMI has been positively correlated to the frequency of fast-food meals consumed. ${ }^{18}$

Among the most important predictors for childhood OW/OB is hyperphagia and reduced physical activity, with numerous studies in Greek children associating excess in body weight with surplus in the energy and fat intake. ${ }^{53,54}$ Since this increased energy intake is usually concomitant to a reduced physical activity level, Greek normal-weight children are found to exhibit less sedentary activities..$^{55}$ Likewise, Greek girls participating in more than 3 hours of extracurricular sport activities are 59\% less likely to be OW/ $\mathrm{OB}$ than their non-participating counterparts. ${ }^{56}$

A plethora of research has evaluated the effect of the family environment on the weight status of children. Parental and children's BMI appear to be directly correlated, ${ }^{24,25,30,57,58}$ based on the authority of this unanimous finding, Lean ${ }^{59}$ suggested that in order to combat pediatric OB one would have to "shrink a parent". Family status is equally important in pediatric OW in Greece, as a significant association was noted between family divorce and children's OW. ${ }^{57} \mathrm{On}$ the other hand, high parental education and a smaller number of children in the family have been shown to inversely affect a child's BMI..$^{57,58}$ Additionally, Greek grandmothers appear to be overfeeding their grandchildren, as higher odds for childhood OW/OB (OR:1.38) has been demonstrated in Greek families where grandmothers are the child's primary caregivers. ${ }^{30,60}$ Economic factors such as residence ownership and annual family income of $12,000-20,000 €$ have been found to increase the odds for childhood OW/OB, while Greek children with excess in body weight are more likely to receive more pocket-money compared to their non-overweight peers. ${ }^{60,61}$

According to Naukkarinen, ${ }^{62}$ the majority of research on the causes and consequences of acquired $\mathrm{OB}$ is encumbered by the incomplete ability to control for genetic influences. What is the actual weight of nature versus nurture and why do researchers focus only on the latter? The independent genetic predispositions associated with $\mathrm{OB}$ are difficult to disentangle in humans. ${ }^{62}$ Additionally, OB treatment is mainly based on behavioral approaches. Thus, we are in need of evidence on the environmental aspects of the condition in order to be able to design interventions. Kafatos ${ }^{63}$ designed a school-based "Health and Nutrition Education" program and demonstrated that it had positive long-term effects on the BMI of Greek school children. According to Manios, ${ }^{64}$ such programs have a positive effect on the level of physical activity and also mediate the level of serum lipids of the participating children. In any case, a holistic approach is needed in order to engage in this public 
health discourse and thereby fruitfully combat the childhood obesity epidemic.

Study limitations of the present report include the great heterogeneity in the samples of the retrieved studies regarding size, origination, age range and methods of data collection. In order to explore the source of heterogeneity, a number of subgroup analyses have been performed, while on a second level sensitivity analyses, after controlling for methodological and epidemiological issues of studies, have been applied. Research using large samples like the Tambalis ${ }^{6}$ study inevitably produced bias in the results. Important strengths of the present study include the conduct of systematic review, the endorsement of the quality metric scale system and the application of sensitivity analyses in the entire body of data and within subgroups as well.

The present study showed that due to the easy application of the IOTF criteria, a plethora of low quality studies have been published, each suggesting a different pediatric prevalence of $\mathrm{OW} / \mathrm{OB}$ in Greece. Due to this, readers need to apply caution and should not extrapolate findings. Frequent systematic reviews are required in order to define the accurate weight status of Greek children, while continuous research is essential so as to actualize and to clarify the trend of excess in body weight in Greek children. Moreover, it would be of great interest to demonstrate the extent to which the economic crisis has had an effect on the disease of abundance, obesity.

\section{CONFLICT OF INTEREST}

This paper was conducted in the framework of the first author's Thesis. The study was supported by the Research Committee of the Aristotle University of Thessaloniki, in the Research Project "Postgraduate Course Adolescent Medicine". (Project code 83023, Supervisor: Ass. Professor Assimina GalliTsinopoulou). The work was presented at the 50th Greek Pediatric Congress and was awarded the "S. Doxiadis Prize" for "Social Pediatrics".

\section{REFERENCES}

1. World Health Organization, 2005 Preventing chronic diseases: A vital investment. World Global Report.
2. Wang Y, Lobstein T, 2006 Worldwide trends in childhood overweight and obesity. Int J Pediatr Obes 1: 11-25.

3. Franks PW, Hanson RL, Knowler WC, et al, 2010 Childhood obesity, other cardiovascular risk factors, and premature death. N Engl J Med 362: 485-493.

4. Wang G, Dietz WH, 2002 Economic burden of obesity in youths aged 6 to 17 years: 1979-1999 Pediatrics 109: 81-91.

5. Lien N, Henriksen HB, Nymoen LL, Wind M, Klepp KI, 2010 Availability of data assessing the prevalence and trends of overweight and obesity among European adolescents. Public Health Nutr 13: 1680-1687.

6. Tambalis KD, Panagiotakos DB, Kavouras SA, et al, 2010 Eleven-year prevalence trends of obesity in Greek children: first evidence that prevalence of obesity is leveling off. Obesity (Silver Spring) 18: 161-166.

7. Haidich AB, 2010 Meta-analysis in medical research. Hippokratia 14: 29-37.

8. Petretta M, Pirozzi F, Sasso L, Paglia A, Bonaduce D, 2011 Review and metaanalysis of the Frequency of Familial Dilated Cardiomyopathy. Am J Cardiol 108: 1171-1176.

9. Chen JJ, Yu CB, Du WB, Li LJ, 2011 Prevalence of hepatitis B and C in HIV-infected patients: a meta-analysis. Hepatobiliary Pancreat Dis Int 10: 122-127.

10. Stroup DF, Berlin JA, Morton SC, et al, 2000 Metaanalysis of observational studies in epidemiology: a proposal for reporting. Meta-analysis Of Observational Studies in Epidemiology (MOOSE) group. JAMA 283: 2008-2012.

11. Cole TJ, Bellizzi MC, Flegal KM, Dietz WH, 2000 Establishing a standard definition for child overweight and obesity worldwide: international survey. BMJ 320 : 1240-1243.

12. Wells GA, Shea B, O'Connell D, et al, The NewcastleOttawa Scale (NOS) for assessing the quality of nonrandomized studies in meta-analyses. [12 May 2011]: Available from: http://www.ohri.ca/programs/clinical_epidemiology/oxford.htm

13. Hardy RJ, Thompson SG, 1998 Detecting and describing heterogeneity in meta-analysis. Stat Med 17: 841-856.

14. Higgins JP, Thompson SG, 2002 Quantifying heterogeneity in a meta-analysis. Stat Med 21: 1539-1558.

15. DerSimonian R, Laird N, 1986 Meta-analysis in clinical trials. Control Clin Trials 7: 177-188.

16. Krassas GE, Tsametis C, Baleki V, et al, 2004 Prevalence of overweight and obesity among children and adolescents in Thessaloniki-Greece and Kayseri-Turkey. Pediatr Endocrinol Rev 1: 460-464.

17. Angelopoulos PD, Manios J, Milionis HJ, 2005 Risk factors for obesity in elementary school children in the Greek border region of Thesprotia. Paediatriki 68: 433-438.

18. Angelopoulos PD, Milionis HJ, Moschonis G, Manios Y, 2006 Relations between obesity and hypertension: preliminary data from a cross-sectional study in primary schoolchildren: the children study. Eur J Clin Nutr 60: 
1226-1234.

19. Christodoulos AD, Flouris AD, Tokmakidis SP, 2006 Obesity and physical fitness of pre-adolescent children during the academic year and the summer period: effects of organized physical activity. J Child Health Care 10: 199-212.

20. Magkos F, Manios Y, Christakis G, Kafatos AG, 2006 Age-dependent changes in body size of Greek boys from 1982 to 2002. Obesity (Silver Spring) 14: 289-294.

21. Magkos F, Piperkou I, Manios Y, et al, 2006 Diet, blood lipid profile and physical activity patterns in primary school children from a semi-rural area of Greece. J Hum Nutr Diet 19: 101-112.

22. Papadimitriou A, Kounadi D, Konstantinidou M, Xepapadaki P, Nicolaidou P, 2006 Prevalence of obesity in elementary schoolchildren living in Northeast Attica, Greece. Obesity (Silver Spring) 14: 1113-1117.

23. Tokmakidis SP, Kasambalis A, Christodoulos AD, 2006 Fitness levels of Greek primary schoolchildren in relationship to overweight and obesity. Eur J Pediatr 165: 867-874.

24. Vlachopapadopoulou E, Karachaliou F, Papadopoulou N, Tsarmaklis G, Michalacos S, 2006 Obesity prevalence in children of elementary schools of the district of Attica, Greece. Ann Clin Paetr 53: 47-53.

25. Manios Y, Costarelli V, Kolotourou M, Kondakis K, Tzavara C, Moschonis G, 2007 Prevalence of obesity in preschool Greek children, in relation to parental characteristics and region of residence. BMC Public Health 7: 178.

26. Christodoulos A, Douda H, Tousoulis D, Tokmakidis S, 2008 The relation of body mass index with selected behavioural parameters in 11 year-old children. Paediatriki 71: 368-375.

27. Kamtsios S, Digelidis N, 2008 Physical activity levels, exercise attitudes, self-perceptions and BMI type of 11 to 12-year-old children. J Child Health Care 12: 232-240.

28. Linardakis M, Sarri K, Pateraki MS, Sbokos M, Kafatos A, 2008 Sugar-added beverages consumption among kindergarten children of Crete: effects on nutritional status and risk of obesity. BMC Public Health 8: 279.

29. Panagiotakos DB, Papadimitriou A, Anthracopoulos MB, et al, 2008 Birthweight, breast-feeding, parental weight and prevalence of obesity in schoolchildren aged 10-12 years, in Greece; the Physical Activity, Nutrition and Allergies in Children Examined in Athens (PANACEA) study. Pediatr Int 50: 563-568.

30. Hassapidou M, Papadopoulou SK, Frossinis A, Kaklamanos I, Tzotzas T, 2009 Sociodemographic, ethnic and dietary factors associated with childhood obesity in Thessaloniki, Northern Greece. Hormones (Athens) 8: 53-59.

31. Kollias AK, Skliros EA, Leotsakos N, Gikas A, Garifallos D, 2009 Childhood obesity in relation to parental weight status in Greece. Hippokratia 13: 253.

32. Koroni M, Garagouni-Areou F, Roussi-Vergou CJ, Za- firopoulou M, Piperakis SM, 2009 The stigmatization of obesity in children. A survey in Greek elementary schools. Appetite 52: 241-244.

33. Mantzouranis N, Douda H, Pilianidis T, Tokmakidis S, 2009 Obesity prevalence in children aged 8-13 years according to IOTF cutoffs, CDC growth charts and ZBMI scores. Paediatriki 72: 41-45.

34. Mavrakanas TA, Konsoula G, Patsonis I, Merkouris BP, 2009 Childhood obesity and elevated blood pressure in a rural population of northern Greece. Rural Remote Health 9: 1150.

35. Chaniotis DI, Botsari S, Mikelopoulou P, Chaniotis F, 2010 Eating habits and obesity indices assessment in School Health based survey in the Attica basin Greek pupils: 2002-2007. e-Journal of Science \& Technology 5: 49-61.

36. Kontogianni MD, Farmaki AE, Vidra N, Sofrona S, Magkanari F, Yannakoulia M, 2010 Associations between lifestyle patterns and body mass index in a sample of Greek children and adolescents. J Am Diet Assoc 110: 215-221.

37. Manios Y, Angelopoulos PD, Kourlaba G, et al, 2010 Prevalence of obesity and body mass index correlates in a representative sample of Cretan school children. Int J Pediatr Obes 6: 135-141.

38. Moschonis G, Tanagra S, Vandorou A, et al, 2010 Social, economic and demographic correlates of overweight and obesity in primary-school children: preliminary data from the Healthy Growth Study. Public Health Nutr 13: 1693-1700.

39. Yannakoulia M, Ntalla I, Papoutsakis C, Farmaki AE, Dedoussis GV, 2010 Consumption of vegetables, cooked meals, and eating dinner is negatively associated with overweight status in children. J Pediatr 157: 815-820.

40. James PT, Leach R, Kalamara E, Shayeghi M, 2001 The Worldwide Obesity Epidemic. Obes Res 9: 228-233.

41. Rokholm B, Baker JL, Sørensen TI, 2010 The levelling off of the obesity epidemic since the year 1999 - a review of evidence and perspectives. Obes Rev 11: 835-846.

42. Manios Y, Magkos F, Christakis G, Kafatos AG, 2005 Changing relationships of obesity and dyslipidemia in Greek children: 1982-2002. Prev Med 41: 846-851.

43. Georgiadis G, Nassis GP, 2007 Prevalence of overweight and obesity in a national representative sample of Greek children and adolescents. Eur J Clin Nutr 61: 1072-1074.

44. Olsen LW, Baker JL, Holst C, Sørensen TI, 2006 Birth cohort effect on the obesity epidemic in Denmark. Epidemiology 17: 292-295.

45. Magarey AM, Daniels LA, Boulton TJ, Cockington RA, 2003 Predicting obesity in early adulthood from childhood and parental obesity. Int J Obes Relat Metab Disord 27: 505-513.

46. Moraeus L, Lissner L, Yngve A, Poortvliet E, Al-Ansari U, Sjöberg, 2012 Multi-level influences on childhood obesity in Sweden: societal factors, parental determinants 
and child's lifestyle. Int J Obes (Lond) 36: 969-976.

47. Júlíusson PB, Eide GE, Roelants M, Waaler PE, Hauspie R, Bjerknes R, 2010 Overweight and obesity in Norwegian children: prevalence and socio-demographic risk factors. Acta Paediatr 99: 900-905.

48. Stamatakis E, Wardle J, Cole TJ, 2010 Childhood obesity and overweight prevalence trends in England: evidence for growing socioeconomic disparities. Int J Obes (Lond) 34: 41-47.

49. Valdés-Pizarro J, Royo-Bordonada MA, 2012 Prevalence of childhood obesity in Spain: National Health Survey 2006-2007. Nutr Hosp 27: 154-160.

50. Pedrosa C, Correia F, Seabra D, Oliveira BM, SimõesPereira C, Vaz-de-Almeida MD, 2011 Prevalence of overweight and obesity among 7-9-year-old children in Aveiro, Portugal: comparison between IOTF and CDC references. Public Health Nutr 14: 14-19.

51. Brambilla P, Vezzoni M, Lucchini R, et al, 2012 Is the prevalence of overweight reducing at age 5-6 years? Ten years data collection in ASL Milano 2. Ital J Pediatr 38: 24.

52. Skelton JA, Irby MB, Grzywacz JG, Miller G, 2011 Etiologies of obesity in children: nature and nurture. Pediatr Clin North Am 58: 1333-1354.

53. Papandreou D, Malindretos P, Rousso I, 2008 Investigation of dietary intake and obesity status in a pediatric population from Northern Greece. Nutr Food Sci 38: 526-533.

54. Manios Y, Grammatikaki E, Papoutsou S, Liarigkovinos T, Kondaki K, Moschonis G, 2008 Nutrient intakes of toddlers and preschoolers in Greece: the GENESIS study. J Am Diet Assoc 108: 357-361.

55. Papoutsakis C, Vidra NV, Hatzopoulou I, et al, 2007 The Gene-Diet Attica investigation on childhood obesity (GENDAI): overview of the study design. Clin Chem Lab Med 45: 309-315.
56. Antonogeorgos G, Papadimitriou A, Panagiotakos DB, Priftis KN, Nicolaidou P, 2011 Association of extracurricular sports participation with obesity in Greek children. J Sports Med Phys Fitness 51: 121-127.

57. Yannakoulia M, Papanikolaou K, Hatzopoulou I, Efstathiou E, Papoutsakis C, Dedoussis GV, 2008 Association between family divorce and children's BMI and meal patterns: the GENDAI Study. Obesity (Silver Spring) 16: 1382-1387.

58. Krassas GE, Tzotzas T, Tsametis C, Konstantinidis T, 2001 Prevalence and trends in overweight and obesity among children and adolescents in Thessaloniki, Greece. J Pediatr Endocrinol Metab 14: 1319-1326.

59. Lean MEJ, 2010 Childhood obesity: time to shrink a parent. Int J Obes 34: 1-3.

60. Moschonis G, Iatridi V, Mavrogianni C, et al, 2011 Accuracy and correlates of visual and verbal instruments assessing maternal perceptions of children's weight status: the Healthy Growth Study. Public Health Nutr 14: 1979-1987.

61. Grammatikopoulou MG, Galli-Tsinopoulou A, Daskalou E, et al, 2008 Is pocket money an indicant of childhood obesity? Arch Dis Child 93: pw4.

62. Naukkarinen J, Rissanen A, Kaprio J, Pietiläinen KH, 2011 Causes and consequences of obesity: the contribution of recent twin studies. Int J Obes Epub. doi: 10.1038/ijo.2011.192

63. Kafatos A, Manios Y, Moschandreas J, Preventive Medicine \& Nutrition Clinic University of Crete Research Team, 2005 Health and nutrition education in primary schools of Crete: follow-up changes in body mass index and overweight status. Eur J Clin Nutr 59: 1090-1092.

64. Manios Y, Kafatos A, 2006 Health and nutrition education in primary schools in Crete: 10 years follow-up of serum lipids, physical activity and macronutrient intake. Br J Nutr 95: 568-575. 